\title{
La responsabilité sociale des facultés de médecine, notion émergente: quelles implications pratiques?
}

\section{Axel Max Klohn, \\ Philippe Chastonay ${ }^{a, b}$}

a Institut de Médecine Sociale et Préventive, Département de Médecine Communautaire, Faculté de Médecine, Université de Genève

b Unité de Développement et de Recherche en Éducation Médicale, Faculté de Médecine, Université de Genève

\section{Correspondance:}

Dr Axel M. Klohn

Institut de Médecine Sociale et Préventive, Département de Médecine et Santé Communautaire, Faculté de Médecine, Université de Genève 1 , rue Michel-Servet $\mathrm{CH}-1211$ Genève 4 Tél. 0787444648 Fax 0223795912

axel.klohn@unige.ch

\section{Résumé}

Contexte: Les profondes mutations technologiques et sociales des dernières décennies, le dilemme d'attribution de ressources limitées, les modifications de rôles entre secteur public et privé, le souci d'adapter l'éducation supérieure aux besoins réels, ont placé la responsabilité sociale des facultés de médecine au centre des préoccupations.

Buts: Nous explorons ici les tensions et synergies entre le concept de responsabilité sociale et d'autres objets de la réforme des études médicales, et présentons des exemples de mise en œuvre concrète.

Thématique: La responsabilité ou imputabilité sociale puise ses sources dans le «contrat social» qui relie chaque institution - et les professionnels qu'elle forme - à la communauté où elle est insérée et puise ses ressources. Elle est complémentaire aux notions de professionalisme et d'efficience, et s'inscrit dans le même cadre éthique. Renforcer l'imputabilité sociale implique un processus d'ouverture à la communauté à de nombreux niveaux, notamment en ce qui concerne ses besoins de santé prioritaires. Les initiatives tendant à renforcer l'imputabilité sociale démontrent une grande synergie avec certains objectifs de la réforme des études medicales, de sorte qu'elles sont appelées à assumer un rôle central dans le dispositif pédagogique. Un cadre conceptuel éprouvé permet d'évaluer le degré d'engagement d'une institution dans ce domaine selon ses quatre dimensions principales: pertinence, qualité, efficacité, équité.

Conclusion: L'emphase sur les services à la communauté de la part des facultés de médecine et la pertinence des enseignements en termes de santé publique paraît de plus en plus prioritaire aussi bien pour stimuler le développement du professionalisme chez les étudiants que pour la survie, le développement et l'indépendance des facultés de médecine: une chance à saisir pour un mutuel bénéfice?

\section{Responsabilité sociale: essai de définition}

La réflexion sur les liens entre facultés de médecine et communauté se développe depuis fort longtemps, mais ce n'est qu'au cours des dernières décennies que ce processus s'est formalisé.

La société et les facultés de médecine sont reliées par un ensemble d'obligations mutuelles [1]: pour la première, le devoir d'assurer les moyens et la liberté nécessaires aux facultés dans la poursuite de leur mission, pour les secondes, un devoir de transparence et une implication avec et dans la communauté. Ce dernier aspect est reconnu par l'Association Médicale Mondiale [2] lorsqu'elle déclare qu'une «faculté de médecine doit réviser fréquemment le cursus proposé afin de tenir compte des besoins de la communauté et des conseils des médecins en exercice».

L'Organisation Mondiale de la Santé a défini la responsabilité sociale des facultés de médecine [3] comme «l'obligation d'orienter leurs activités d'éducation, de recherche et de services vers la satisfaction de questions de santé prioritaires dans la communauté, région et/ou nation qu'elles ont le mandat de servir; ces questions de santé prioritaires sont définies de façon conjointe par les gouvernements, les organisations sanitaires, les professionnels de santé et le public». En d'autres termes, la responsabilité sociale en éducation médicale passe nécessairement par un développement de la dimension communautaire [4].

\section{Responsabilité sociale ou la recherche de la pertinence, la qualité, I'efficacité et l'équité}

Si la responsabilité sociale des écoles de médecine implique une ouverture accrue et de plus grandes interactions avec la communauté et les réseaux, y compris dans la planification et la conduite de la recherche, comment avancer dans ce processus? Sur le plan conceptuel, nous devons une percée importante à une initiative de l'OMS dans les années 90 qui a jetté les bases pour mieux quantifier et codifier une notion longtemps perçue comme floue et rhétorique. La monographie de Boelen et Heck [3] analyse la responsabilité sociale des institutions de santé selon quatre dimensions principales: pertinence, qualité, efficacité, équité. Une balance doit être négociée entre celles-ci avec la participation de la communauté. Différentes cultures et contraintes aboutissent ainsi à des solutions différentes. Ces dimensions, et tout particulièrement «la capacité à anticiper et répondre aux besoins et attentes du public» [5] en proposant des services orientés vers les besoins prioritaires des populations, sont à la racine même de l'impact de l'éducation médicale et des institutions de formation, ainsi que de leurs possibilités de survie dans un environnement de plus en plus difficile. 


\section{Responsabilité sociale des facultés de médecine: quelles conséquences pour I'enseignement?}

Il existe en parallèle de cette réflexion de responsabilité sociale institutionnelle une réflexion sur ce qu'il correspond d'enseigner aux étudiants en termes de responsabilité sociale au niveau individuel. Faulkner et McCurdy [6] définissent la responsabilité sociale individuelle comme l'aptitude à s'engager dans «des activités qui contribuent au bonheur, à la santé et à la prospérité d'une communauté et de ses membres».

\section{Responsabilité sociale des facultés de médecine et professionalisme?}

Former des professionels sérieux et socialement responsables à un niveau individuel constitue seulement une partie des défis auxquels doivent faire face les facultés de médecine en termes de responsabilité sociale: de fait il s'agit en premier lieu de positionner l'institution d'enseignement dans ses rapports avec la communauté. En effet, l'acquisition des valeurs du professionalisme par les étudiants se voit renforcée dans la mesure où, «pour comprendre la portée de leur rôle social, ils doivent voir [les valeurs] démon-

\section{«Les enseignants sont-ils convaincus qu'il est important d'enseigner la responsabilité sociale aux étudiants?»}

L'immersion des étudiants dans la communauté pour des activités de formation pourrait jouer ici un rôle favorable: les expériences de pratique précoce, développées dans la majorité des cas dans un environnement communautaire [7], semblent à même de renforcer un processus contrôlé de «socialisation à la profession» [8] et un ensemble de traits positifs tels que la satisfaction des étudiants, leur niveau de confiance, et leurs choix d'engagement avec les besoins de la communauté [7].

Est-ce pour autant suffisant? «Placer les étudiants dans des situations communutaires est certes une activité louable, mais insuffisante pour garantir la responsabilité sociale. Nous avons besoin aujourd'hui d'étendre l'acceptation du mandat d'apprentissage centré sur la communauté, soutenu par un financement bien ciblé des initiatives d'éducation et de recherche» [4]. Il s'agit de «renforcer l'intensité et la pertinence de la formation aux soins de premier niveau, étendre le curriculum au-delà de sa focalisation bio-

\section{«Les initiatives tendant à renforcer la responsabilité sociale démontrent une grande synergie avec certains objectifs de la réforme des études médicales»}

médicale, encourager la recherche sur les systèmes de santé, et évaluer l'impact des politiques sociales dans l'amélioration de la santé des populations» [9]. Le défi est de «transformer l'agenda de recherche de façon à inclure des domaines qui n'ont pas été jusqu'ici au centre des intérêts des chercheurs académiques, tout en conservant le leadership dans les aires traditionnelles de recherche» [10]. trées par leurs écoles de médecine, leurs doyens, leurs universités» [10]. La responsabilité sociale des facultés de médecine - interprétée au niveau institutionnel - apparaît ainsi comme un instrument de première importance dans le dispositif pédagogique destiné à transmettre les valeurs du professionalisme et de responsabilité sociale au niveau individuel.

De nos jours, le préambule de la charte du professionalisme médical l'affirme encore: «le professionalisme est la base du contrat entre la médecine et la société» [11]. Cette notion s'est cependant vue soumise à rude épreuve un peu partout [12] au point qu'un recadrage plus large du concept de professionalisme a pu sembler nécessaire de fraîche date: «le professionalisme ne se compose pas seulement d'expertise, de compétences techniques et d'éthique. Il inclut d'autres dimensions significatives, non cognitives, telles que des valeurs, un esprit de service, une mission, la faculté d'établir une relation thérapeutique. Inculquer et renforcer le professionalisme peut demander une approche différente que d'enseigner l'expertise professionnelle» [13].

\section{Evaluer I'engagement d'une faculté de médecine en termes de responsabilité sociale} «Pour l'institution de formation, la responsabilité sociale devrait être appréciée à l'aide de normes relevant de trois domaines interdépendants: la conception, la production et l'utilisabilité des personnels de santé» [14]. En pratique, il s'agit en tout premier lieu, de répertorier les activités existantes, souvent instaurées de façon informelle au fil du temps, et de leur conférer une expression plus formelle, unifiée et explicite de façon à mieux les mettre en valeur et «poser les jalons de parténariats pour la santé respectueux avec le gouvernement, les autorités de santé, les communautés et les entreprises» [10]. Au niveau institutionnel, le cadre conceptuel de Boelen et Heck [3] nous 
fournit une application immédiate sous la forme d'une grille qui peut être employée pour l'auto-évaluation des facultés de médecine (Tableau 1).

Woollard [15] suggère un complément intéressant pour une telle évaluation avec des critères, inspirés par Richards et coll. [16], concernant l'orientation communautaire d'une faculté de médecine:

- Quel est le degré auquel les grands principes de base de l'école sont orientés vers la communauté?

- Quelle emphase est mise dans le curriculum sur la connaissance et les concepts de ce qu'est une communauté et une population? Comment mesurer et répondre à des besoins de santé? Comment prendre en compte le contexte social et culturel de manière appropriée?

- A quel degré le curriculum intègre-t-il l'apprentissage en immersion communautaire?

- Quel est le degré d'implication de la communauté dans le programme de formation?

- Quels sont les liens organiques entre la faculté ou le programme et le système de santé?

En sus, Faulkner et McCurdy [5] proposent plusieurs «questions importantes» à prendre en considération pour la conception d'un cursus spécifique:

- Les enseignants sont-ils convaincus qu'il est important d'enseigner la responsabilité sociale aux étudiants?

- Existe-t-il une disposition favorable pour conférer à un cursus consacré à la responsabilité sociale un rang comparable à celui des autres composants du programme d'éducation médicale?

- Quel sera le processus employé pour le développement des objectifs éducatifs du cursus?

- Comment les écoles de médecine peuvent-elles identifier les candidats qui seront réceptifs à ce cursus? (en particulier si l'enseignement est longitudinal et reparti sur plusieurs années)

- Quels seminaires et quelle didactique seront-ils abordés dans le cursus?

- Quels types d'expériences d'apprentissage sontelles les plus à même d'aider les étudiants à atteindre les objectifs éducationnels du cursus?

- Comment identifier et préparer les enseignants et tuteurs qui vont enseigner, superviser et guider les étudiants lors du cursus?

\section{Soutien de la société à I'engagement social d'une faculté de médecine}

Le soutien du politique semble important dans le renforcement de l'engagement social d'une faculté de médecine. A titre d'exemple citons le Canada. Dans ce pays, qui assume un rôle pionnier dans l'initiative de l'OMS «Unité pour la santé» [17], un document de position sur la responsabilité sociale des facultés de médecine a été développé dès 2001 par Santé Canada [18]. Un fonds spécifique, le Fonds pour l'adaptation des soins de santé primaires de Santé, a financé des initiatives renforçant l'engagement social des facultés de médecine du pays. Quelques-uns de ces projets sont répertoriés dans le Tableau 2. On y retrouve des objectifs tels que la création de réseau, le soutien à la création et à l'évaluation de modèles de bonnes pratiques, et le renforcement de synergies entre professionnels de santé, mais aussi des initiatives relatives à la santé des populations minoritaires, à risque ou négligées.

Lors d'une mise à jour récente [19], l'AFMC met en exergue des activités en rapport avec le recrutement et la formation des étudiants aborigènes, un réseau de travail sur l'enseignement en santé publique, un programme de formation professorale destiné à ceux qui enseignent aux diplômés hors Canada et EtatsUnis, un groupe de travail sur la formation des futurs médecins dans les soins palliatifs et de fin de vie, et une initiative concernant l'accréditation de programmes de formation interprofessionnels. Il existe en outre une base de données en ligne de projets se rapportant à la responsabilité sociale, comportant 145 projets à l'heure actuelle [20], un site contenant des informations sur le professionalisme, et un forum de partenariat.

L'expérience canadienne est ainsi l'une des mieux documentées dans le domaine. Plus récemment, un cadre général a été proposé, permettant de définir des normes, critères spécifiques et indicateurs de pertinence sociale [14]. A l'heure où d'autres universités francophones relèvent le défi, il se pourrait qu'un engagement explicite dans une politique de responsabilité sociale avec recherche d'impact sur la santé de la société devienne un jour un critère d'importance dans l'accréditation et l'attribution d'un label d'excellence pour les facultés de médecine [14].

Tableau 1

Grille d'évaluation de la responsabilité sociale pour les institutions de santé selon Boelen et Heck [3].

\begin{tabular}{llll}
\multicolumn{1}{c}{$\begin{array}{l}\text { domaines } \\
\text { éducation }\end{array}$} & recherche & services \\
\hline Dimensions & planification actions impact planification actions impact planification actions impact \\
\hline Pertinence & & & \\
\hline Qualité & & & \\
\hline Efficacité & & & \\
\hline Equité & & &
\end{tabular}




\section{Tableau 2}

Quelques initiatives de facultés de médecine orientées vers le renforcement de leur responsabilité sociale

\begin{tabular}{|c|c|}
\hline & source \\
\hline $\begin{array}{l}\text { Développement et coordination d'une unité de médecine rurale } \\
\text { intégrant un modèle communautaire de soins }\end{array}$ & Parboosingh 2003 [10] \\
\hline $\begin{array}{l}\text { Développement d'un modèle d'éducation médicale } \\
\text { basé sur la communauté }\end{array}$ & Parboosingh 2003 [10] \\
\hline $\begin{array}{l}\text { Initiatives se rapportant a recrutement et à la formation } \\
\text { d'étudiants aborigènes }\end{array}$ & AFMC 2002 [20] \\
\hline Initiatives se rapportant aux besoins de santé des minorités & AFMC 2002 [20] \\
\hline $\begin{array}{l}\text { Détermination de bonnes pratiques d'enseignement } \\
\text { de la santé publique }\end{array}$ & AFMC 2002 [20] \\
\hline $\begin{array}{l}\text { Détermination de bonnes pratiques d'enseignement sur les soins } \\
\text { palliatifs et de fin de vie }\end{array}$ & AFMC 2002 [20] \\
\hline $\begin{array}{l}\text { Développement et évaluation de programmes d'enseignement } \\
\text { interprofessionnels }\end{array}$ & AFMC 2002 [20] \\
\hline
\end{tabular}

\section{Conclusion}

L'emphase sur les services à la communauté de la part des facultés de médecine et la pertinence des enseignements en termes de santé publique apparaissent de plus en plus prioritaires aussi bien pour stimuler le développement du professionalisme chez les étudiants que pour la survie, le développement et l'indépendance des facultés de médecine: une chance à saisir pour un mutuel bénéfice?

\section{Références}

1 Pellegrino E. Academic health centres in society: an ethical reflection. Acad Med. 1999;74:21-6.

2 Association Médicale Mondiale. Prise de Position de l'AMM sur la Formation Médicale adoptée par l'Assemblée générale de l'AMM, Pilanesberg, Afrique du Sud, octobre 2006. (Consulté le 13.1.2009). http://www.wma.net/f/policy/e15.htm

3 Boelen C, Heck J. Defining and measuring the social accountability of medical schools, Geneva: World Health Organization; 1995.

4 Hennen B. Demonstrating social accountability in medical education. CMAJ. 1997;156(3):365-7.

5 Boelen C. L'éducation médicale vue par un expert international en santé publique. Pédagogie Médicale. 2007;8(1):44-50.

6 Faulkner L, McCurdy R. Teaching Medical Students Social Responsibility: The Right Thing to Do. Acad Med. 2000;75(4):346-50.
7 Littlewood S, Ypinazar V, Margolis S, Scherpbier A, Spencer J, Dornan T. Early practical experience and the social responsiveness of clinical education: systematic review. BMJ. 2005;331(7513):387-91.

8 Dornan T, Bundy C. What can experience add to early medical education? Consensus survey. BMJ. 2004;329(Clinical research ed.):834

9 Peabody J. Measuring the social responsiveness of medical schools: setting the standards. Acad Med. 1999;74(8 Suppl):S59-S68.

10 Parboosingh J. Medical schools' social contract: more than just education and research. CMAJ. 2003;168(7):852-3.

11 ABIM Foundation, American Board of Internal Medicine, ACP-ASIM Foundation, America College of Physicians-American Society of Internal Medicine, European Federation of Internal Medicine. Medical professionalism in the new millennium: a physician charter. Ann Intern Med. 2002; 136(3):243-6.

12 Cruess R, Cruess S, Johnston S. Renewing professionalism: an opportunity for medicine. Academic Medicine. 1999;74(7):878-84.

13 Remen R, Rabow M. The Healer's Art: professionalism, service and mission. Medical Education. 2005;39(10):1167-8.

14 Boelen C, Grand'Maison P, Ladner J, Pestiaux D. Responsabilité sociale et accréditation. Une nouvelle frontière pour l'institution de formation. Pédagogie Médicale. 2008;9(4):235-44.

15 Woollard R. Caring for a common future: medical school's social accountability. Med Educ. 2006;40(4):301-13.

16 Richards R, Fulop T, Bannerman J. Innovative Schools for Health Personnel: Report on Ten Schools Belonging to the Network of Community Oriented Educational Institutions for Health Sciences. Geneva: World Health Organisation; 1987.

17 Boelen C. Vers l'unité pour la santé: défis et opportunités des partenariats pour le développement de la santé (P Grand'Maison \& Bolduc, Trad.) Geneva: World Health Organisation; 2002

18 Santé Canada. Imputabilité sociale. Une vision pour les facultés de médecine du Canada. Ottawa (Ontario): Santé Canada; 2001.

19 Maskill S. Update on AFMC social accountability initiatives. AFMC FORUM. 2007;40(2):19.

20 Association des facultés de médecine du Canada. Base de données sur les initiatives en matière d'imputabilité sociale. (Consulté le 22.12.2009). www.afmc.ca/social-initiatives-database-f.php 